\section{Síndrome de Burnout em Médicos de Estratégia Saúde da Família de Montes Claros, MG, e Fatores Associados}

\author{
Burnout Syndrome in Family Health Strategy Physicians in \\ Montes Claros, MG, and Associated Factors \\ Síndrome de Burnout en Médicos de la Estrategia Salud de la \\ Familia de Montes Claros, MG, y Factores Asociados
}

\section{Resumo}

Objetivo: O estudo objetivou investigar a prevalência da síndrome de Burnout em médicos da Estratégia Saúde da Família em Montes Claros, MG, e sua associação com estresse no trabalho, desequilíbrio esforço-recompensa e qualidade de vida. Métodos: Foi realizada uma pesquisa epidemiológica, quantitativa, telematizada, analítica e transversal. O questionário autoaplicável Maslach Burnout Inventory foi aplicado a 89 médicos para diagnóstico da síndrome. Os médicos também proveram dados sociodemográficos e preencheram os instrumentos Job Stress Scale, Escala Desequilíbrio-Esforço-Recompensa e WHOQOL-BREF para identificação de condições de trabalho. As variáveis categóricas foram analisadas por distribuição de frequências. $A$ associação entre variáveis de fatores de risco e presença de síndrome de Burnout foi realizada pelo teste de Qui-quadrado de Pearson. Resultados: Houve $100 \%$ de prevalência da síndrome de Burnout moderada nos médicos avaliados. Os escores das dimensões Exaustão e Despersonalização, constituintes do instrumento avaliativo da síndrome de Burnout, tiveram correlação positiva com alta demanda psicológica e profissional, alto desgaste e esforço, comprometimento excessivo ao trabalho e desbalanço na razão esforço-recompensa. Eles também se relacionaram a atributos de qualidade de vida, tais quais má condição física, social e ambiental. Conclusão: Medidas preventivas e interventivas devem ser tomadas para a diminuição do estresse laboral dos médicos avaliados, assim reduzindo a ocorrência da síndrome de Burnout.

Palavras-chave: Esgotamento Profissional; Saúde da Família; Atenção Primária à Saúde; Qualidade de Vida; Saúde do Trabalhador
Ariadna Janice Drumond Morais ${ }^{a}$

Cristiane Borborema Teles ${ }^{b}$

Laryssa Ferreira Rochab

Marise Fagundes Silveira ${ }^{a}$

Lucinéia de Pinho ${ }^{\circ}$

a Programa de Pós-Graduação em

Cuidado Primário em Saúde, Universidade

Estadual de Montes Claros (UNIMONTES).

Montes Claros, MG, Brasil.

ariadnajanice@live.co.uk;

ciaestatistica@yahoo.com.br

b Universidade Estadual de Montes Claros (UNIMONTES). Montes Claros, MG, Brasil. cris_borborema@yahoo.com.br; laryssarochha@gmail.com

c Universidade Estadual de Montes Claros (UNIMONTES); Faculdades Integradas Pitágoras de Montes Claros (FIP-MOC). Montes Claros, MG, Brasil. lucineiapinho@ hotmail.com (Autora correspondente)

Fonte de financiamento: declaram não haver.

Parecer CEP:

762.251 (UNIMONTES), aprovado em 22/08/2014.

Conflito de interesses: declaram não haver.

Procedência e revisão por pares: revisado por pares.

Recebido em: 14/05/2018.

Aprovado em: 01/08/2018. 


\begin{abstract}
Objective: The study aimed to investigate the prevalence of Burnout syndrome in physicians of the Family Heath Strategy at Montes Claros, MG, and the association with work stress, effort-reward imbalance and quality of life. Methods: Epidemiologic, quantitative, web-based, analytical and cross-sectional research. The self-administered questionnaire Maslach Burnout Inventory was applied to 89 physicians to diagnose the syndrome. The physicians also provided sociodemographic data and responded the Job Stress Scale, the Imbalance-Effort-Reward scale and the WHOQOL-BREF for identification of work conditions. The categoric variables were analyzed by frequency distribution. The association between variables of risk factors and the presence of Burnout syndrome was tested by the Pearson's chi-square test. Results: $100 \%$ of the physicians evaluated exhibited moderate Burnout syndrome. The scores for the Exhaustion and Depersonalization dimensions, which are components of the inventory for Burnout Syndrome assessment, were positively correlated with high psychological and professional demands, high strain and effort, excess commitment to the job and imbalanced effort-reward ratio. They were also associated with attributes of quality of life such as poor physical, social and environmental conditions. Conclusion: Preventive and intervention actions are required to decrease work-related stress of the evaluated physicians, thereby decreasing Burnout syndrome prevalence.
\end{abstract}

Keywords: Burnout, Professional; Family Health; Primary Health Care; Quality of Life; Occupational Health

\title{
Resumen
}

Objetivo: El estudio objetivó investigar la prevalencia del síndrome de Burnout en médicos de la Estrategia Salud de la Familia en Montes Claros, MG, y su asociación con estrés en el trabajo, desequilibrio esfuerzo-recompensa y calidad de vida. Métodos: Se realizó una investigación epidemiológica, cuantitativa, por medio de la web, analítica y transversal. El cuestionario autoaplicable Maslach Burnout Inventory se aplicó a 89 médicos para el diagnóstico del síndrome. Los médicos también proporcionaron datos sociodemográficos y llenaron los instrumentos Job Stress Scale, Escala Desequilibrio-Esfuerzo-Recompensa y WHOQOL-BREF para identificación de condiciones de trabajo. Las variables categóricas fueron analizadas por distribución de frecuencias. La asociación entre variables de factores de riesgo y presencia de síndrome de Burnout fue realizada por la prueba de chi-cuadrado de Pearson. Resultados: Hubo un $100 \%$ de prevalencia de la Síndrome de Burnout moderada en los médicos evaluados. Los escores de las dimensiones Agotamiento y Despersonalización, constituyentes del instrumento evaluativo del síndrome de Burnout, tuvieron correlación positiva con alta demanda psicológica y profesional, alto desgaste y esfuerzo, comprometimiento excesivo al trabajo y desbalance en la razón esfuerzo-recompensa. También se relacionaron con atributos de calidad de vida tales como mala condición física, social y ambiental. Conclusión: Se deben tomar medidas preventivas y de intervenciones para disminuir el estrés laboral de los médicos evaluados, reduciendo así la incidencia del síndrome de Burnout.

Palabras clave: Agotamiento Profesional; Salud de la Familia; Atención Primaria de Salud; Calidad de Vida; Salud Laboral

\section{Introdução}

O estresse no trabalho é um conjunto de reações físicas e emocionais prejudiciais que ocorrem quando as pressões ou exigências do labor não se igualam à capacidade, aos recursos ou às necessidades do trabalhador. Essas reações devem ser tratadas com seriedade, uma vez que podem causar doenças psicofisiológicas no trabalhador. ${ }^{1}$

O estresse crônico ocupacional é conhecido como síndrome do Esgotamento Profissional ou síndrome de Burnout. ${ }^{2}$ O termo Burnout foi utilizado pela primeira vez pelo psicólogo Freudenberger, em 1974, para definir um estado de exaustão física e mental ligado ao trabalho. ${ }^{2}$ É uma síndrome que produz um estado de fadiga ou frustração crônico relacionado à condição emocional das pessoas em relação ao seu trabalho, modo de vida ou relacionamento e que ocorre devido à dedicação intensa a uma causa, que não produz o resultado esperado. ${ }^{3}$ Sendo um grave problema de caráter psicossocial, ela vem afetando trabalhadores em todo mundo.

O estresse ocupacional pode ser desencadeado pela ação conjunta de fatores intrínsecos ao trabalho e seu ambiente, como a falta de comunicação, o estilo de gestão, interferências burocráticas, ausência 
de participação na tomada de decisões, dificuldade nas relações interpessoais ou ausência de uma política favorável à vida familiar. ${ }^{1,4-6}$ Estes fatores atuando a longo prazo contribuem para o adoecimento e aparecimento do estresse crônico. ${ }^{1,4-6}$

A SB manifesta-se através das sintomatologias física, comportamental, psíquica e defensiva. ${ }^{7}$ Os sintomas apresentados são: fadiga constante, distúrbios do sono, falta de atenção, alterações da memória, ansiedade, negligência no trabalho, irritabilidade, baixa concentração, relações interpessoais conflituosas, absenteísmo, tendência ao isolamento e sentimento de impotência. ${ }^{1,3,7}$ A sobrecarga psíquica e tensional dos trabalhadores acometidos pelo Burnout é significante e eles deveriam ser periodicamente avaliados para recuperação e preservação da sua saúde mental e física. ${ }^{2,8}$

O esgotamento crônico também acomete profissionais da área da saúde, incluindo aqueles que atuam na Estratégia de Saúde da Família (ESF). ${ }^{5,9,10}$ Este serviço é um dos principais pilares do SUS e caracteriza-se em expansão em todo o território nacional. Nos últimos anos a cobertura da população pela Saúde da Família no Brasil evoluiu de 50,9\% (2008) para 53,4\% (2013). ${ }^{11}$ A boa saúde física e emocional dos profissionais na ESF, entre eles o médico, é um fator que pode impactar na qualidade do serviço. ${ }^{9}$

No contexto da ESF, os médicos estão expostos a diversos desafios e estressores laborais e, caso não utilizem estratégias de enfrentamento adequadas, ficam vulneráveis ao esgotamento profissional. ${ }^{5,6} \mathrm{O}$ resultado é a baixa qualidade de atendimento, o aumento de erros médicos, processos judiciais e diminuição da capacidade de expressar empatia. ${ }^{4,6}$

O Burnout é um problema que pode comprometer não apenas o trabalhador, mas também os usuários ESF. Apesar disso, essa afecção ainda é pouco conhecida, merecendo ser investigada por seu número, seriedade potencial e domínios que atinge. ${ }^{10} \mathrm{~A}$ investigação da SB em trabalhadores da saúde como integrantes do ESF é essencial para o diagnóstico precoce e planejamento de medidas preventivas e de promoção da saúde dos mesmos. ${ }^{2,6}$ Nesse sentido, o presente estudo investigou a prevalência da síndrome de Burnout em médicos de ESF no município de Montes Claros, MG, e avaliou sua associação com estresse no trabalho, desequilíbrio esforço-recompensa e qualidade de vida.

\section{Métodos}

\section{Caracterização do estudo e da população}

Trata-se de um estudo epidemiológico, quantitativo, telematizado, analítico e transversal de levantamento de dados. Foi desenvolvido no município de Montes Claros, MG, situado na mesorregião do norte de Minas Gerais. O município apresenta Índice de Desenvolvimento Humano Municipal (IDH-M) médio de 0,770 e índice de Gini de 0,5391. À época do estudo, o município possuía 135 equipes de ESF, todas incluídas no Programa Nacional de Melhoria do Acesso e da Qualidade da Atenção Básica (PMAQ-AB).

A população da pesquisa compreendeu todos os 122 médicos das equipes de ESF que atuavam na área urbana do município. Não foram incluídos médicos de zona rural, estrangeiros, com carga horária inferior a 40 horas e os afastados do trabalho. Entre os convidados para participar da pesquisa, 89 responderam os questionários. 


\section{Instrumentos e coleta de dados}

Os dados foram coletados entre outubro de 2015 a fevereiro de 2016, por meio de um questionário online, enviado por e-mail aos profissionais. O questionário contemplava a avaliação da síndrome de Burnout - variável dependente, e as características sociodemográficas, ocupacionais e os fatores de riscos - variáveis independentes.

O perfil sociodemográfico e ocupacional foi avaliado por meio das variáveis sexo, idade, estado civil, escolaridade e horas de trabalho. A síndrome de Burnout foi avaliada por meio do instrumento Maslach Burnout Inventory, constituído por 22 itens, que englobam as dimensões exaustão emocional (9 itens); despersonalização (5 itens) e realização profissional (8 itens). ${ }^{3,12} \mathrm{~A}$ forma de pontuação dos itens pesquisados adota a escala do tipo Likert que varia de zero a seis: (0) nunca, (1) uma vez ao ano ou menos, (2) uma vez ao mês ou menos, (3) algumas vezes no mês, (4) uma vez por semana, (5) algumas vezes por semana e (6) todos os dias. O escore do sujeito é computado pelo somatório dos pontos dos itens relativos a cada uma das dimensões.

Para o cálculo da síndrome de Burnout, foram utilizados os pontos de corte do estudo original de Maslach, ${ }^{3}$ com a avaliação dos três domínios que compõem a síndrome. ${ }^{12}$ A dimensão "exaustão emocional" acima dos 27 pontos é considerada um nível de burnout elevado. Quando existem valores entre 16-26 é indicador de níveis médios e abaixo de 16 corresponde a níveis de burnout baixos. Para o quesito de "despersonalização", as pontuações superiores a 13 representam níveis altos, de 7-12 moderado e inferior a 6 indica um nível baixo de burnout. Por fim, a "realização profissional" é oposta as dimensões anteriores, portanto, níveis maiores ou iguais a 39 pontos representam um acometimento baixo, entre 32 a 38 pontos um nível moderado e menor ou igual a 31 é um nível alto de burnout. Neste estudo, a presença de nível alto nas dimensões "exaustão emocional" e "despersonalização" e baixas pontuações em "realização profissional" caracterizou a síndrome de Burnout.

Para investigar as fontes geradoras de estresse e suas repercussões na saúde e nas relações sociais no ambiente de trabalho, utilizou-se a Job Stress Scale, criada por Robert Karasek, ${ }^{13}$ que propôs um modelo teórico bidimensional que relaciona dois aspectos (demandas e controle no trabalho) ao risco de adoecimento. ${ }^{14}$ A versão reduzida da Job Stress Scale, elaborada por Töres Theorell em 1988 e adotada neste trabalho, é composta por 17 questões divididas em 3 dimensões: demanda psicológica (5 itens), controle (6 itens) e apoio social ( 6 itens). ${ }^{14}$

A forma de pontuação dos itens do instrumento Job Stress Scale adota a escala do tipo Likert (1-4), variando entre "frequentemente" e "nunca/quase nunca", ou entre "concordo totalmente" e "discordo totalmente". O aspecto demanda tinha como mínimo e máximo possíveis, respectivamente, 5 e 20 pontos, o controle, 6 e 24 pontos e o suporte (apoio social), 6 e 24 pontos". 14,15

Os quadrantes demanda-controle foram definidos a partir da análise das dimensões demanda psicológica e controle sobre o trabalho e adotou-se a média como ponto de corte. Para a dimensão demanda psicológica, o grau "baixa demanda" (abaixo), ou "alta demanda" (maior ou igual à média) e para a dimensão controle o grau "baixo controle" (abaixo da média), ou "alto controle" (maior ou igual à média). A partir das dimensões da demanda (alta e baixa) e do controle (alto e baixo), definiram-se os quadrantes do modelo demanda-controle em alto desgaste no trabalho. 
O modelo foi categorizado em quatro quadrantes: alto desgaste no trabalho (alta demanda psicológica e baixo controle), trabalho ativo (alta demanda psicológica e alto controle), trabalho passivo (baixa demanda psicológica e baixo controle) e baixo desgaste (baixa demanda psicológica e alto controle)".14,15

A avaliação do desequilíbrio entre o esforço realizado e a recompensa recebida no trabalho foi realizada por meio da Escala Desequilíbrio-Esforço-Recompensa desenvolvida por Siegrist. ${ }^{16}$ Ela compreende 23 itens e é composta por três dimensões: esforço (6 itens); recompensa (11 itens) e excesso de comprometimento no trabalho (6 itens). "A escala possui 17 itens com respostas dicotômicas (discordo ou concordo): 6 itens relacionados a esforço extrínseco (com escore total de 5 a 30) e 11 itens relacionados a recompensa (com escore total de 11 a 55).

Ainda, para cada resposta "concordo" ou "discordo", somam-se quatro opções em escala likert (1-4) que variam de "não estressado" a "muito estressado". Os seis itens restantes contêm a dimensão de compromisso excessivo com o trabalho, com respostas em escala likert (1-4), indo do "discordo totalmente" a "concordo totalmente".

O escore de cada dimensão da escala é calculado e utiliza-se a média como ponto de corte para classificação: esforço (alto/baixo), recompensa (alta/baixa), comprometimento excessivo (presente/ausente). O indicador Desequilíbrio Esforço-Recompensa foi calculado considerando a razão entre a média do escore do esforço dividido pela média do escore da recompensa. Os resultados foram categorizados em "equilíbrio" (valores $\leq 1$ ) e "desequilíbrio" (valores $>1$ ). ${ }^{16}$

Para avaliação da qualidade de vida, utilizou-se o questionário WHOQOL-BREF elaborado pelo Grupo de Qualidade de Vida da OMS com 26 questões. ${ }^{17,18}$ A primeira questão refere-se à qualidade de vida de modo geral, e a segunda à satisfação com a própria saúde. As demais questões estão divididas nos domínios: físico (sete itens), psicológico (seis itens), relações sociais (três itens) e meio ambiente (oito itens). ${ }^{17}$

As respostas às questões do WHOQOL-BREF são dadas numa escala likert com um intervalo de 0 (zero) a 5 (cinco), segundo a metodologia Whoqol. A equação sugerida pela OMS foi aplicada para a estimativa de escores de cada domínio. Os escores finais de cada domínio são calculados por uma sintaxe, que considera as respostas de cada questão que compõem o domínio, resultando em escores finais numa escala de 4 a 20". Classificou-se cada um dos domínios do WHOQOL-BREF em satisfatório (maior ou igual à média) e insatisfatório (abaixo da média). ${ }^{17,18}$

\section{Análise dos dados}

As variáveis categóricas foram descritas pela distribuição de frequências. Os escores das dimensões dos instrumentos Maslach Burnout Inventory, Job Stress Scale, Escala Desequilíbrio-Esforço-Recompensa e WHOQOL-BREF foram expressos por estatística descritiva.

Testou-se a associação entre os indicadores da síndrome de Burnout (dimensões de Exaustão e Despersonalização do Maslach Burnout Inventory) e variáveis sociodemográficas e relacionadas ao tempo dedicado ao trabalho por meio do teste não paramétrico de Qui-quadrado de Pearson, com probabilidade de erro de 0,05. 
Foi também estimada a magnitude da associação entre as dimensões Exaustão e Despersonalização do Maslach Burnout Inventory e os outros indicadores (Job Stress Scale, Escala Desequilíbrio-EsforçoRecompensa e WHOQOL-BREF) com uso da regressão logística, adotando-se como medida de associação a razão de chances (OR), com intervalo de 95\% de confiança. Os dados foram analisados utilizando-se o software estatístico Statistical Package for the Social Sciences- SPSS, versão 18.0 para Windows.

\section{Considerações Éticas}

A pesquisa foi aprovada pelo Comitê de Ética em Pesquisa da Unimontes (parecer de nº 762.251 de 15/08/2014), dentro dos parâmetros contidos na Resolução no 466 de 2012 da Comissão Nacional de Ética em Pesquisa do Ministério da Saúde, que estipula normas éticas regulamentadoras de pesquisas envolvendo seres humanos.

Os participantes receberam o Termo de Consentimento Livre e Esclarecido e Gestão Municipal de Montes Claros e assinaram um termo de concordância com a pesquisa. Foram assegurados o anonimato e a confidencialidade dos participantes e das informações, utilizados exclusivamente para fins científicos.

\section{Resultados}

\section{Perfil da População}

Participaram deste estudo 89 médicos da ESF, sendo a maioria do sexo feminino $(71,9 \%)$ e jovem, com idade inferior a 30 anos $(47 \%)$. Aproximadamente metade da população $(49,4 \%)$ era casada ou vivia em união estável. Quanto à formação acadêmica, 52,8\% dos profissionais possuía algum tipo de especialização (Tabela 1).

No relato sobre a jornada de trabalho, $88,8 \%$ dos médicos declararam cumprir até no máximo 40 horas semanais no turno diurno. A maioria não cumpria turno noturno $(65,2 \%)$ e também não trabalhava nos fins de semana, seja em turno diurno $(64,0 \%)$ ou noturno $(84,3 \%)$ (Tabela 1$)$.

\section{Prevalência de síndrome de Burnout e fatores associados}

Entre os médicos da ESF observou-se que 100\% apresentavam nível médio de Burnout. Mais de $60 \%$ relataram altos níveis esgotamento nos domínios "exaustão emocional" e "despersonalização" (Tabela 2).

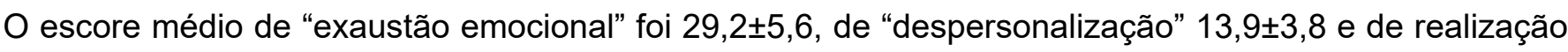
profissional $24,1 \pm 3,6$.

Na caracterização do estresse no trabalho pela Job Stress Scale, destaca-se a "alta demanda psicológica" em quase um terço da população, "baixo controle" em quase metade e "alto desgaste" em $19 \%$ da população. Nos quadrantes do Job Stress Scale, observou-se um predomínio de "baixo desgaste" e de "trabalho passivo" (Tabela 2). O escore médio de "demanda psicológica" foi 16,6 $\pm 2,1$, o de "controle" $19,8 \pm 3,0$ e o de "suporte social" 18,8 $\pm 3,4$.

$\mathrm{Na}$ escala Desequilíbrio-Esforço-Recompensa, as respostas de "alto" e "baixo esforço" para o trabalho e "comprometimento excessivo" não foram relativamente balanceadas. A média de "recompensa" foi classificada como acima da média e a "razão esforço-recompensa" abaixo da média, tendendo 
Tabela 1. Variáveis sóciodemográficas e relacionadas as horas de trabalho dos médicos da Estratégia de Saúde da Família de Montes Claros $(n=89)$.

\begin{tabular}{|c|c|c|}
\hline Variável & $\mathbf{N}$ & $\%$ \\
\hline \multicolumn{3}{|l|}{ Sociodemográficas } \\
\hline \multicolumn{3}{|l|}{ Sexo } \\
\hline Masculino & 25 & 28,1 \\
\hline Feminino & 64 & 71,9 \\
\hline \multicolumn{3}{|l|}{ Idade } \\
\hline Menos de 30 anos & 47 & 52,8 \\
\hline 30 a 39 anos & 31 & 34,8 \\
\hline 40 anos ou mais & 11 & 12,4 \\
\hline \multicolumn{3}{|l|}{ Escolaridade } \\
\hline Especialização & 47 & 52,8 \\
\hline Graduação & 42 & 47,2 \\
\hline \multicolumn{3}{|l|}{ Estado Civil } \\
\hline Casado/União estável & 44 & 49,4 \\
\hline Solteiro/Viúvo/divorciado & 45 & 50,6 \\
\hline \multicolumn{3}{|l|}{ Horas de trabalho } \\
\hline \multicolumn{3}{|l|}{ Horas diurnas semana } \\
\hline $40 \mathrm{~h} / \mathrm{semana}$ & 79 & 88,8 \\
\hline Mais de 40 horas/semana & 10 & 11,2 \\
\hline \multicolumn{3}{|l|}{ Horas noturnas semana } \\
\hline Não trabalha noturno & 58 & 65,2 \\
\hline 1h a 8 h/semana & 11 & 12,3 \\
\hline Mais de 8h/semana & 20 & 22,5 \\
\hline \multicolumn{3}{|c|}{ Trabalho diurno final de semana } \\
\hline Não trabalha & 57 & 64,0 \\
\hline Trabalha & 32 & 36,0 \\
\hline \multicolumn{3}{|c|}{ Trabalho noturno final de semana } \\
\hline Não trabalha & 75 & 84,3 \\
\hline Trabalha & 14 & 15,7 \\
\hline Total & 89 & 100,0 \\
\hline
\end{tabular}

ao desequilíbrio em $43,8 \%$ da população (Tabela 2). O escore médio para "esforço" foi $8,4 \pm 1,3$, para "recompensa" $16,1 \pm 1,9$ e para "excesso de comprometimento" 15,4 2 2,8.

$\mathrm{Na}$ avaliação geral da "qualidade de vida" $55,1 \%$ dos médicos foram classificados como abaixo da média (insatisfatória) nos âmbitos "físico", "social" e de "ambiente", com exceção do domínio "psicológico" (Tabela 2). O escore médio no âmbito "físico" foi 14,8 $\pm 2,6$, no "psicológico" 14,5 $\pm 2,6$, nas "relações pessoais" $14,9 \pm 3,1$, no "ambiente" $14,2 \pm 2,3$ e no "aspecto geral" $14,5 \pm 2,2$. O escore médio de "qualidade de vida" foi autoavaliado em $14,5 \pm 3,7$.

Não houve associação entre os indicadores de Burnout estimados pelo Maslach Burnout Inventory (dimensões "exaustão" e "despersonalização") e variáveis sociodemográficas ou jornada de trabalho (Tabela 3). 
Tabela 2. Respostas de médicos da Estratégia de Saúde da Família de Montes Claros ( $\mathrm{n}=89)$ aos instrumentos Maslach Burnout Inventory, Job Stress Scale e Desequilíbrio-Esforço-Recompensa.

\begin{tabular}{|c|c|c|}
\hline Variáveis & $\mathbf{N}$ & $\%$ \\
\hline \multicolumn{3}{|l|}{ Maslach Burnout Inventory } \\
\hline \multicolumn{3}{|l|}{ Exaustão emocional } \\
\hline Nível Médio & 32 & 36,0 \\
\hline Nível Alto & 57 & 64,0 \\
\hline \multicolumn{3}{|l|}{ Despersonalização } \\
\hline Nível Médio & 35 & 39,3 \\
\hline Nível Alto & 54 & 60,7 \\
\hline \multicolumn{3}{|l|}{ Realização profissional } \\
\hline Nível Médio & 01 & 1,1 \\
\hline Nível Alto & 88 & 98,9 \\
\hline \multicolumn{3}{|l|}{ Síndrome de Burnout } \\
\hline Nível Médio & 89 & 100,0 \\
\hline \multicolumn{3}{|l|}{ Job Stress Scale } \\
\hline \multicolumn{3}{|l|}{ Demanda psicológica } \\
\hline Baixa demanda ( $\downarrow D)$ & 59 & 66,3 \\
\hline Alta demanda $(\uparrow D)$ & 30 & 33,7 \\
\hline \multicolumn{3}{|l|}{ Controle sobre o trabalho } \\
\hline Baixo controle $(\downarrow C)$ & 44 & 49,4 \\
\hline Alto controle $(\uparrow C)$ & 45 & 50,6 \\
\hline \multicolumn{3}{|l|}{ Quadrantes demanda-controle } \\
\hline Baixo desgaste $(\downarrow D \uparrow C)$ & 31 & 34,8 \\
\hline Trabalho passivo $(\downarrow D \downarrow C)$ & 28 & 31,5 \\
\hline Trabalho ativo $(\uparrow D \uparrow C)$ & 13 & 14,6 \\
\hline Alto desgaste $(\uparrow D \downarrow C)$ & 17 & 19,1 \\
\hline \multicolumn{3}{|c|}{ Desequilíbrio-Esforço-Recompensa } \\
\hline \multicolumn{3}{|c|}{ Esforço } \\
\hline Baixo & 40 & 44,9 \\
\hline Alto & 49 & 55,1 \\
\hline \multicolumn{3}{|l|}{ Recompensa } \\
\hline Alta & 50 & 56,2 \\
\hline Baixa & 39 & 43,8 \\
\hline \multicolumn{3}{|l|}{ Comprometimento excessivo } \\
\hline Ausente & 42 & 47,2 \\
\hline Presente & 47 & 52,8 \\
\hline \multicolumn{3}{|l|}{ Razão esforço-recompensa } \\
\hline$\leq 1$ (Equilíbrio) & 50 & 56,2 \\
\hline$>1$ (Desequilíbrio) & 39 & 43,8 \\
\hline \multicolumn{3}{|l|}{ WHOQOL-BREF } \\
\hline \multicolumn{3}{|l|}{ Física } \\
\hline Satisfatória & 43 & 48,3 \\
\hline Insatisfatória & 46 & 51,7 \\
\hline \multicolumn{3}{|l|}{ Psicológica } \\
\hline Satisfatória & 52 & 58,4 \\
\hline Insatisfatória & 37 & 41,6 \\
\hline \multicolumn{3}{|l|}{ Relações Sociais } \\
\hline Satisfatória & 44 & 49,4 \\
\hline Insatisfatória & 45 & 50,6 \\
\hline \multicolumn{3}{|l|}{ Ambiente } \\
\hline Satisfatória & 37 & 41,6 \\
\hline Insatisfatória & 52 & 58,4 \\
\hline \multicolumn{3}{|l|}{ Qualidade de Vida Geral } \\
\hline Satisfatória & 40 & 44,9 \\
\hline Insatisfatória & 49 & 55,1 \\
\hline
\end{tabular}


Tabela 3. Associação entre frequência de Exaustão e Despersonalização com variáveis sociodemográficas e de jornada de trabalho.

\begin{tabular}{|c|c|c|c|c|c|c|}
\hline \multirow{2}{*}{ Variáveis } & \multicolumn{3}{|c|}{ Exaustão (\%) } & \multicolumn{3}{|c|}{ Despersonalização (\%) } \\
\hline & Média & Alta & $\mathbf{p}$ & Média & Alta & p \\
\hline \multicolumn{7}{|l|}{ Sociodemográficas } \\
\hline Sexo & & & 0,323 & & & 0,573 \\
\hline Masculino & 44,0 & 56,0 & & 44,0 & 56,0 & \\
\hline Feminino & 32,8 & 67,2 & & 37,5 & 62,5 & \\
\hline Idade & & & 0,282 & & & 0,194 \\
\hline Menos de 30 anos & 29,8 & 70,2 & & 34,0 & 66,0 & \\
\hline 30 a 39 anos & 38,7 & 61,3 & & 38,7 & 61,3 & \\
\hline 40 anos ou mais & 54,5 & 45,5 & & 63,6 & 36,4 & \\
\hline Escolaridade & & & 0,200 & & & 0,130 \\
\hline Especialização & 29,8 & 70,2 & & 31,9 & 68,1 & \\
\hline Graduação & 42,9 & 57,1 & & 47,6 & 52,4 & \\
\hline Estado Civil & & & 0,937 & & & 0,895 \\
\hline Casado/União estável & 36,4 & 63,9 & & 38,6 & 61,4 & \\
\hline Solteiro/Viúvo/divorciado & 35,6 & 64,4 & & 40,0 & 60,0 & \\
\hline \multicolumn{7}{|l|}{ Horas de trabalho } \\
\hline Diurnas durante a semana & & & 0,366 & & & 0,273 \\
\hline 40 horas & 30,2 & 69,8 & & 45,3 & 54,7 & \\
\hline Mais de 40 horas & 40,0 & 60,0 & & 20,0 & 80,0 & \\
\hline Noturnas durante a semana & & & 0,603 & & & $0,10 s$ \\
\hline Não trabalha & 39,7 & 60,3 & & 46,6 & 53,4 & \\
\hline 1 a 8 horas & 27,3 & 72,7 & & 36,4 & 63,6 & \\
\hline Mais de 8 horas & 30,0 & 70,0 & & 20,0 & 80,0 & \\
\hline Trabalho diurno final de semana & & & 0,488 & & & 0,105 \\
\hline Não trabalha & 38,6 & 61,4 & & 45,6 & 54,4 & \\
\hline Trabalha & 31,2 & 68,8 & & 28,1 & 71,9 & \\
\hline Trabalho noturno final de semana & & & 0,217 & & & 0,135 \\
\hline Não trabalha & 38,7 & 61,3 & & 42,7 & 57,3 & \\
\hline Trabalha & 21,4 & 78,6 & & 21,4 & 78,6 & \\
\hline
\end{tabular}

Na relação entre as dimensões do Maslach Burnout Inventory e parâmetros da Job Stress Scale (Tabela 4), observou-se que foram associados à maior chance de "exaustão" os parâmetros "alta demanda psicológica" e "alto desgaste", ambos com risco acima de $80 \%$. E foram associados à maior chance de "despersonalização" os fatores "alta demanda psicológica", "trabalho ativo" e "alto desgaste", também com risco acima de $80 \%$.

Na associação com os parâmetros da Escala Desequilíbrio-Esforço-Recompensa, maiores chances de "exaustão" e "despersonalização" foram associadas ao esforço maior ou igual à média. A "despersonalização" foi também associada a "comprometimento excessivo". A razão esforço-recompensa maior que 1 foi associada às maiores chances de exaustão e despersonalização entre os profissionais (Tabela 4).

Todos os escores dos domínios e de "qualidade de vida geral" do WHOQOL-BREF mostraram associação significativa com a síndrome de Burnout, nas dimensões "exaustão" e "despersonalização", exceto o domínio "psicológico" em relação à "despersonalização" (Tabela 4). 
Tabela 4. Associação entre as dimensões Exaustão e Despersonalização (Maslach Burnout Inventory) e Estresse no trabalho, Qualidade de Vida e Desequilíbrio Esforço-Recompensa. Montes Claros, MG, 2015.

\begin{tabular}{|c|c|c|c|c|c|c|c|c|}
\hline & \multicolumn{4}{|c|}{ Exaustão } & \multicolumn{4}{|c|}{ Despersonalização } \\
\hline & Média (\%) & Alta (\%) & OR (IC95\%) & $\mathbf{p}$ & Média (\%) & Alta (\%) & OR (IC95\%) & $\mathbf{p}$ \\
\hline \multicolumn{9}{|l|}{ Job Stress Scale } \\
\hline \multicolumn{9}{|l|}{ Demanda psicológica } \\
\hline Baixa demanda $(\downarrow D)$ & 44,1 & 55,9 & 1,0 & & 52,5 & 47,5 & 1,0 & \\
\hline Alta demanda ( $\uparrow \mathrm{D})$ & 20,0 & 80,0 & $3,2(1,1-8,8)$ & 0,025 & 13,3 & 86,7 & $7,2(2,2-23,2)$ & 0,000 \\
\hline \multicolumn{9}{|l|}{ Controle sobre o trabalho } \\
\hline Alto controle $(\uparrow C)$ & 45,5 & 54,5 & 1,0 & & 45,5 & 54,5 & 1,0 & \\
\hline Baixo controle $(\downarrow C)$ & 26,7 & 73,3 & $2,3(0,9-5,6)$ & 0,065 & 33,3 & 66,7 & $1,7(0,8-3,9)$ & 0,242 \\
\hline \multicolumn{9}{|l|}{ Quadrantes demanda-controle } \\
\hline Baixo desgaste ( $\downarrow D \uparrow C)$ & 51,6 & 48,4 & 1,0 & & 58,1 & 41,9 & 1,0 & \\
\hline Trabalho passivo $(\downarrow D \downarrow C)$ & 35,7 & 64,3 & $1,9(0,7-5,5)$ & 0,222 & 46,4 & 53,6 & $1,6(0,6-4,5)$ & 0,373 \\
\hline Trabalho ativo ( $\uparrow \mathrm{D} \uparrow \mathrm{C})$ & 30,8 & 69,2 & $2,4(0,7-9,5)$ & 0,211 & 15,4 & 84,6 & $7,6(1,4-40,3)$ & 0,017 \\
\hline Alto desgaste $(\uparrow D \downarrow C)$ & 11,8 & 88,2 & $8,0(1,6-41,0)$ & 0,013 & 11,8 & 88,2 & $10,4(2,0-53,5)$ & 0,005 \\
\hline \multicolumn{9}{|c|}{ Desequilíbrio-Esforço-Recompensa (DER) } \\
\hline \multicolumn{9}{|l|}{ Esforço } \\
\hline Baixo & 55,0 & 45,0 & 1,0 & & 62,5 & 37,5 & 1,0 & \\
\hline Alto & 20,4 & 79,6 & $4,8(1,9-12,2)$ & 0,001 & 20,4 & 79,6 & $6,5(2,5-16,7)$ & 0,000 \\
\hline \multicolumn{9}{|l|}{ Recompensa } \\
\hline Alta & 25,6 & 74,4 & 1,0 & & 28,2 & 71,8 & 1,0 & \\
\hline Baixa & 44,0 & 56,0 & $0,4(0,2-1,1)$ & 0,073 & 48,0 & 52,0 & $0,4(0,2-1,0)$ & 0,058 \\
\hline \multicolumn{9}{|l|}{ Comprometimento excessivo } \\
\hline Ausente & 44,7 & 55,3 & 1,0 & & 57,4 & 42,6 & 1,0 & \\
\hline Presente & 26,2 & 73,8 & $2,3(0,9-5,6)$ & 0,070 & 19,0 & 81,0 & $5,7(2,2-14,9)$ & 0,000 \\
\hline \multicolumn{9}{|l|}{ Razão esforço-recompensa } \\
\hline$\leq 1$ (Equilíbrio) & 22,0 & 78,0 & 1,0 & & 22,0 & 78,0 & 1,0 & \\
\hline >1 (Desequilíbrio) & 53,8 & 46,2 & $0,2(0,1-0,6)$ & 0,002 & 61,5 & 38,5 & $0,2(0,1-0,4)$ & 0,000 \\
\hline \multicolumn{9}{|l|}{ WHOQOL-BREF } \\
\hline \multicolumn{9}{|l|}{ Física } \\
\hline Satisfatória & 55,8 & 44,2 & 1,0 & & 62,8 & 37,2 & 1,0 & \\
\hline Insatisfatória & 17,4 & 82,6 & $6,0(2,3-15,8)$ & 0,000 & 17,4 & 82,6 & $8,0(3,0-21,4)$ & 0,000 \\
\hline \multicolumn{9}{|l|}{ Psicológica } \\
\hline Satisfatória & 48,1 & 51,9 & 1,0 & & 46,2 & 53,8 & 1,0 & \\
\hline Insatisfatória & 18,9 & 81,1 & $3,9(1,5-10,6)$ & 0,005 & 29,7 & 70,3 & $2,0(0,8-4,9)$ & 0,118 \\
\hline \multicolumn{9}{|l|}{ Relações Sociais } \\
\hline Satisfatória & 54,5 & 45,5 & 1,0 & & 50,0 & 50,0 & 1,0 & \\
\hline Insatisfatória & 17,8 & 82,2 & $5,6(2,1-14,6)$ & 0,000 & 28,9 & 71,1 & $2,5(1,1-5,9)$ & 0,042 \\
\hline \multicolumn{9}{|l|}{ Ambiente } \\
\hline Satisfatória & 51,4 & 48,6 & 1,0 & & 56,8 & 43,2 & 1,0 & \\
\hline Insatisfatória & 25,0 & 75,0 & $3,2(1,3-7,8)$ & 0,011 & 26,9 & 73,1 & $3,6(1,5-8,7)$ & 0,005 \\
\hline \multicolumn{9}{|l|}{ Qualidade de Vida Geral } \\
\hline Satisfatória & 55,0 & 45,0 & 1,0 & & 57,5 & 42,5 & 1,0 & \\
\hline Insatisfatória & 20,4 & 79,6 & $4,8(1,9-12,1)$ & 0,001 & 24,5 & 75,5 & $4,2(1,7-10,3)$ & 0,002 \\
\hline
\end{tabular}

As diferenças significativas $(p<0.05)$ são destacadas em negrito. 


\section{Discussão}

\section{Resumo dos principais achados do estudo}

Neste estudo os médicos da ESF do município de Montes Claros, MG, avaliados apresentaram a síndrome de Burnout em um grau moderado. Os indicadores da síndrome de Burnout (dimensões "exaustão" e "despersonalização") foram associados com alta demanda psicológica e profissional, alto desgaste e esforço, comprometimento excessivo ao trabalho e desbalanço na razão esforço-recompensa. Os atributos de qualidade de vida também foram associados à síndrome de Burnout. Não foi observada associação entre os indicadores de Burnout e variáveis sociodemográficas e de jornada de trabalho dos profissionais.

\section{Fortalezas e limitações do estudo}

Este estudo contribuiu para avaliação da saúde mental de médicos no cenário da ESF. Os resultados obtidos podem subsidiar medidas para promoção da saúde do trabalhador no município. Os resultados devem ser considerados à luz de algumas limitações. O delineamento transversal impossibilita a relação de causalidade entre as variáveis. Deve-se considerar o contexto local de atuação das equipes de ESF, o que dificulta a generalização dos resultados. As perdas de profissionais podem ter influenciado os resultados finais e subestimado a prevalência de sofrimento ocupacional encontrada, ainda que tenha sido alta.

\section{Comparação com a literatura já existente}

As taxas de prevalência da síndrome de Burnout em médicos de família na literatura variam entre $34,8 \%$ a $85,7 \% .^{5,11,19-21} \mathrm{Em}$ um estudo nos Estados Unidos foi observado que mais que $50 \%$ dos médicos especialistas em medicina de família apresentaram a síndrome de Burnout. ${ }^{20} \mathrm{Em}$ Portugal, a prevalência de Burnout foi 46,9\% nos residentes de Medicina Geral e Familiar (MGF). ${ }^{21}$ No Brasil, em avaliação da síndrome de Burnout em Profissionais da Estratégia Saúde da Família, detectou-se em 56,67\% dos médicos. ${ }^{10}$ No presente estudo foi observado $100 \%$ dos médicos acometidos com Burnout em grau moderado. Além disso, os médicos apresentaram altos percentuais de risco para "exaustão emocional" e "despersonalização", o que pode comprometer a qualidade dos serviços prestados e no vínculo desses profissionais com a comunidade.

A causa do desenvolvimento da síndrome de Burnout é multifatorial e está relacionada a fatores tanto individuais quanto laborais. ${ }^{1,3}$ No presente estudo os dados demográficos não foram associados à síndrome de Burnout. Dos Santos et al. ${ }^{21}$ e Cubillo et al. ${ }^{19}$ em Portugal e Espanha, respectivamente, também mostraram a ausência da correlação entre dados demográficos e a síndrome. Já Martins et al. ${ }^{10}$ e Silva et al. ${ }^{22}$, no Brasil, ao avaliarem os profissionais vinculados à Atenção Primária à Saúde, verificaram que aqueles mais jovens possuíam maior risco da síndrome de Burnout.

Entre as características laborais, a jornada de trabalho pode ter influência na síndrome de Burnout, visto que turnos com mais de 48 horas por semana expõem os trabalhadores a riscos potenciais à saúde. ${ }^{23}$ No entanto, $88,8 \%$ da população estudada trabalhava dentro do limite de 40 horas semanais, e não houve relação desse fator com o desenvolvimento da síndrome. A jornada de trabalho na ESF com limite de 
40 horas semanais ou menos é recomendada ${ }^{24}$ e adotada em vários países, ${ }^{23}$ podendo inclusive ser um fator protetor ao aparecimento da síndrome de Burnout. ${ }^{5}$

Observou-se também, um índice significativo de "trabalho passivo" obtido nas avaliações pela Job Stress Scale (28\% da população), o que junto ao alto índice de "comprometimento excessivo", expresso pela escala Desequilíbrio-Esforço-Recompensa (47\%), sugere que os profissionais avaliados adotam um modelo de trabalho chamado "taylorismo".

Esse modelo se baseia na repetição de tarefas, divisão das mesmas entre formuladores e executores, estabelecimento de metas de produtividade, dificuldade na comunicação entre os diferentes níveis hierárquicos e cobrança entre pares..$^{25,26} \mathrm{Com}$ a regulação do desempenho dos médicos, o taylorismo pode gerar um sentimento de frustração e impotência nos profissionais. ${ }^{19,27}$

Pelo Job Stress Scale detectou-se que aproximadamente um terço da população exibia "alta demanda psicológica" e 19\% reportou "alto desgaste", o que merece atenção, visto a associação dessas variáveis com "exaustão" e "despersonalização". Essa relação corrobora outros estudos que alegam que o ambiente de ESF é um agente estressor.

Os médicos estressados e emocionalmente exaustos sentem-se sobrecarregados e mais frustrados com seus empregos. ${ }^{6}$ Reforçando a sobrecarga dos médicos, os indicadores da síndrome de Burnout também foram associados ao "esforço acima da média" e "comprometimento excessivo", detectados pelo Desequilíbrio-Esforço-Recompensa. Alguns desses fatores aumentaram em mais de 8 vezes a chance de "exaustão" e mais de 10 vezes a chance de "despersonalização".

Os dados de "razão esforço-recompensa" do Desequilíbrio-Esforço-Recompensa também se associaram aos parâmetros da síndrome de Burnout, mostrando o impacto do desequilíbrio entre o grande esforço despendido no trabalho e a baixa recompensa recebida. A desvalorização econômica do profissional, insatisfação com as atividades assistenciais, a perda de prestígio frente à sociedade, a insegurança em relação ao vínculo com a ESF, associadas à dependência de interesses político, altas cargas assistenciais e a pressão excessiva também são explorados em outros estudos como fatores desencadeadores de insatisfação, baixa realização profissional e estresse laboral. ${ }^{27,29}$

No quesito qualidade de vida apenas o aspecto "psicológico" foi reportado como satisfatório, e praticamente todos os itens foram associados com os parâmetros de "exaustão" e "despersonalização". Esses resultados reforçam que o modelo de trabalho adotado pelos médicos da ESF não Ihes assegura uma boa qualidade de vida. Pode-se inferir que conciliar relações familiares/sociais, domínio físico e ambiente com relações de trabalho é difícil para a maioria desses profissionais, pois o estresse interfere na qualidade de vida, sendo prejudicial à vida familiar e trabalhista. ${ }^{28}$

A ESF tem atraído jovens médicos, sem experiência profissional, a maioria com idade inferior a 30 anos, como uma opção para a inserção no mercado de trabalho apesar dos baixos salários, falta de infraestrutura, riscos biológicos e problemas relativos à organização do trabalho. ${ }^{19,30} \mathrm{O}$ fato do jovem profissional ter que lidar com essas dificuldades laborais, enquanto falha em atingir suas altas expectativas, concretizar anseios, idealizações e desejos, promove o aparecimento do estresse profissional. ${ }^{6}$ Apesar do alto esforço desprendido pelo profissional para obter resultados satisfatórios, muitas vezes ele se defronta com limitações institucionais, que levam a um sentimento de baixa realização profissional, desapontamento e desgaste..$^{5,31}$ 
Outro fator que pode influenciar o estresse laboral dos médicos na ESF é o modelo utilizado na organização do trabalho, via Programa Nacional de Melhoria do Acesso e da Qualidade da Atenção Básica (PMAQ-AB). ${ }^{26} \mathrm{O}$ programa define as atribuições dos médicos e os força a uma busca desenfreada por resultados, numa tentativa de introjeção de missão e valores. ${ }^{21,25}$ Embora o objetivo do PMAQ-AB seja a satisfação dos usuários do serviço, ele não garante uma estrutura sólida para sua realização e pode, em alguns casos, ser uma forma de prescrição do trabalho em saúde. Sugerem-se em estudos futuros a avaliação deste programa no estresse profissional.

\section{Implicações para a pesquisa na área e/ou para a prática dos profissionais}

As condições de saúde mental do médico na ESF podem repercutir na qualidade dos serviços prestados à população. O profissional acometido pelo Burnout fica desmotivado para seu trabalho, o que acarreta em perda da qualidade da assistência no campo da saúde pública. ${ }^{7} \mathrm{O}$ esgotamento profissional dificulta o processo de tomada de decisão clínica, resultando em ineficiência da gestão das despesas farmacêuticas e laboratoriais. ${ }^{4,6}$ Além disso, a exaustão os torna mais insensíveis e aumenta as chances de abandono no trabalho. 5,6

Conforme determinado na Política Nacional de Saúde do Trabalhador, os gestores devem desenvolver estratégias para identificar situações de risco ou produção de agravos à saúde do trabalhador, adotando medidas de controle quando necessário. ${ }^{21,32}$ No Brasil, a síndrome é considerada risco ocupacional e é contemplada em leis federais de auxílio ao trabalhador, no campo de transtornos mentais e do comportamentos relacionados ao trabalho. ${ }^{33}$

\section{Conclusões}

Os resultados indicam que todos os médicos da ESF de Montes Claros avaliados apresentavam a síndrome de Burnout em um grau moderado. Os principais fatores associados à síndrome foram alta demanda psicológica e profissional, alto desgaste e esforço, comprometimento excessivo ao trabalho e desbalanço na razão esforço-recompensa, além de atributos de qualidade de vida como condições físicas, sociais e ambientais.

Medidas preventivas e interventivas devem ser tomadas para a diminuição do estresse laboral dos médicos para prevenir a ocorrência do Burnout e permitir um melhor desempenho profissional. Para melhor conhecimento do tema, são necessárias outras investigações, avaliando novos contextos, pois esses estudos são escassos. Uma abordagem qualitativa das questões expostas pode ser útil para apreender significados e percepções dos sujeitos acometidos pela síndrome.

\section{Referências}

1. National Institute for Occupational Safety and Health. STRESS...At Work; 1999 [cited 2017 Apr 18]. Available from: http://www.cdc.gov/ niosh/docs/99-101/

2. Trigo TR, Teng CT, Hallak JEC. Síndrome de Burnout ou estafa profissional e os transtornos psiquiátricos. Rev Psiq Clín. 2007;34(5):22333. DOI: http://dx.doi.org/10.1590/S0101-60832007000500004

3. Maslach C, Jackson SE. The measurement of experienced burnout. J Occup Behav. 1981;2:99-113. DOI: http://dx.doi.org/10.1002/ job.4030020205 
4. Kushnir T, Greenberg D, Madjar N, Hadari I, Yermiahu Y, Bachner YG. Is burnout associated with referral rates among primary care physicians in community clinics? Fam Pract. 2014;31(1):44-50.

5. Morelli, SG, Sapede M, Silva ATC. Burnout em médicos da Atenção Primária: uma revisão sistemática. Rev Bras Med Fam Comunidade. 2015;10(34):1-9. DOI: http://dx.doi.org/10.5712/rbmfc10(34)958

6. Trindade LL, Lautert L. Síndrome de Burnout entre os trabalhadores da Estratégia de Saúde da Família. Rev Esc Enferm USP. 2010;44(2):274-9. DOI: http://dx.doi.org/10.1590/S0080-62342010000200005

7. Benevides-Pereira AMT. O Estado da Arte do Burnout no Brasil. Rev Eletrônica InterAção Psy. 2003;1(1):68-75.

8. Freire PL, Trentin JP, de Avila-Quevedo L. Trends in burnout syndrome and emotional factors: an assessment of anesthesiologists in Southern Brazil, 2012. Psychol Health Med. 2016;21(4):413-23. DOI: http://dx.doi.org/10.1080/13548506.2016.1139143

9. Albuquerque FJB, Melo CF, Araújo Neto JL. Avaliação da síndrome de Burnout em profissionais da Estratégia Saúde da Família da capital paraibana. Psicol Reflex Crit. 2012;25(3):542-9. DOI: http://dx.doi.org/10.1590/S0102-79722012000300014

10. Martins LF, Laport TJ, Menezes VP, Medeiros PB, Ronzani TM. Esgotamento entre profissionais da Atenção primária à Saúde. Ciênc Saúde Coletiva. 2014;19(12):4739-50. DOI: http://dx.doi.org/10.1590/1413-812320141912.03202013

11. Malta DC, Santos MAS, Stopa SR, Vieira JEB, Melo EA, Reis AAC. A Cobertura da Estratégia de Saúde da Família (ESF) no Brasil, segundo a Pesquisa Nacional de Saúde, 2013. Ciênc Saúde Coletiva. 2016;21(2):327-38. DOI: http://dx.doi.org/10.1590/141381232015212.23602015

12. Carlotto MS, Câmara SG. Propriedades psicométricas do Maslach Burnout Inventory em uma amostra multifuncional. Estud Psicol (Campinas). 2007;24(3):325-32. DOI: http://dx.doi.org/10.1590/S0103-166X2007000300004

13. Karasek RA Jr. Job Demands, Job Decision Latitude, and Mental Strain: Implications for Job Redesign. Adm Sci Q. 1979;24(2):285-308. DOI: http://dx.doi.org/10.2307/2392498

14. Alves MGM, Chor D, Faerstein E, Lopes CS, Werneck GL. Versão resumida da "job stress scale”: adaptação para o português. Rev Saúde Pública. 2004;38(2):164-71. DOI: http://dx.doi.org/10.1590/S0034-89102004000200003

15. Urbanetto JS, Silva PC, Hoffmeister E, de Negri BS, da Costa BEP, Figueiredo CEP. Workplace stress in nursing workers from an emergency hospital: Job Stress Scale analysis. Rev Latino-Am Enferm. 2011;19(5):1122-31. DOI: http://dx.doi.org/10.1590/S010411692011000500009

16. Siegrist J, Marmot M. Health inequalities and the psychosocial environment-two scientific challenges. Soc Sci Med. 2004;58(8):1463-73. DOI: http://dx.doi.org/10.1016/S0277-9536(03)00349-6

17. The World Health Organization Quality of Life Assessment (WHOQOL): development and general psychometric properties. Soc Sci Med. 1998;46(12):1569-85. DOI: http://dx.doi.org/10.1016/S0277-9536(98)00009-4

18. Fleck MPA, Louzada S, Xavier M, Chachamovich E, Vieira G, Santos L, et al. Aplicação da versão em português do instrumento abreviado de avaliação da qualidade de vida "WHOQOL-BREF”. Rev Saúde Pública. 2000;34(2):178-83. DOI: http://dx.doi.org/10.1590/S003489102000000200012

19. Cubillo ACM, Guevara JC, Bravo JJM, Riguera MJP, Castro MLG, Sanz AG. Evolución del burnout y variables asociadas em los médicos de atención primaria. Aten Primaria. 2012;44(9):532-9.

20. Shanafelt TD, Boone S, Tan L, Dyrbye LN, Sotile W, Satele D, et al. Burnout and satisfaction with work-life balance among US physicians relative to the general US population. Arch Intern Med. 2012;172(18):1377-85. DOI: http://dx.doi.org/10.1001/archinternmed.2012.3199

21. Dos Santos SCR, Viegas AIF, Morgado CIMO, Ramos CSV, Soares CND, Roxo HMCJ, et al. Prevalência de burnout em médicos residentes de Medicina Geral e Familiar em Portugal. Rev Bras Med Fam Comunidade. 2017;12(39):1-9. DOI: http://dx.doi.org/10.5712/ rbmfc12(39) 1430

22. Silva SCPS, Nunes MAP, Santana VR, Reis FP, Machado Neto J, Lima SO. A síndrome de burnout em profissionais da Rede de Atenção Primária à Saúde de Aracaju, Brasil. Ciênc Saúde Coletiva. 2015;20(10):3011-20. DOI: http://dx.doi.org/10.1590/1413812320152010.19912014

23. Lee S, McCann D, Messenger JC. Duração do trabalho em todo o mundo: tendências de jornadas de trabalho, legislação políticas numa perspectiva global comparada. Brasília: Organização Internacional do Trabalho; 2009.

24. Brasil. Ministério da Saúde. Portaria no 2.436, de 21 de setembro de 2017. Aprova a Política Nacional de Atenção Básica, estabelecendo a revisão de diretrizes para a organização da Atenção Básica, no âmbito do Sistema Único de Saúde (SUS). Brasília: Diário Oficial da União; 2017. 
25. Moraes PN, Iguti AM. Avaliação do desempenho do trabalhador como forma peculiar de prescrição do trabalho: uma análise do PMAQAB. Saúde Debate. 2013;37(98):416-26.

26. Shimizu HE, Carvalho-Junior DA. O processo de trabalho na Estratégia Saúde da Família e suas repercussões no processo saúdedoença. Ciênc Saúde Coletiva. 2012;17(9):2405-14. DOI: http://dx.doi.org/10.1590/S1413-81232012000900021

27. Feliciano KVO, Kovacs MH, Sarinho SW. Burnout entre médicos da saúde da família: os desafios da transformação do trabalho. Ciênc Saúde Coletiva. 2011;16(8):3373-82. DOI: http://dx.doi.org/10.1590/S1413-81232011000900004

28. Marcelino G, Cerveira JM, Carvalho I, Costa JA, Lopes M, Calado NE, et al. Burnout levels among Portuguese Family doctors: a nationwide survey. BMJ Open. 2012;2(3). pii: e001050. DOI: http://dx.doi.org/10.1136/bmjopen-2012-001050

29. Gonçalves CR, Cruz MT, Oliveira MP, Morais AJD, Moreira KS, Rodrigues CAQ, et al. Recursos humanos: fator crítico para as redes de atenção à saúde. Saúde Debate. 2014;38(100):26-34.

30. Carreiro GSP, Ferreira Filha MO, Lazarte R, Silva AO, Dias MD. O processo de adoecimento mental do trabalhador da Estratégia Saúde da Família. Rev Eletrônica Enferm. 2013;15(1):146-55.

31. Miranzi SSC, Mendes CA, Nunes AA, Iwamoto HH, Miranzi MAS, Tavares DMS. Qualidade de vida e perfil sociodemográfico de médicos da estratégia de saúde da família. Rev Med Minas Gerais. 2010;20(2):189-97.

32. Brasil. Ministério da Saúde. Portaria no 1.823, de 23 de agosto de 2012. Institui a Política Nacional de Saúde do Trabalhador e da Trabalhadora. Brasília: Diário Oficial da União; 2012.

33. Brasil. Presidência da República. Casa Civil. Subchefia para Assuntos Jurídicos. Lei no 8.213, de 24 de julho de 1991 . Dispõe sobre os Planos de Benefícios da Previdência Social e dá outras providências. Brasília: Diário Oficial da União; 1991. 